\title{
Decentralized Recommendation based on Matrix Factorization: A Comparison of Gossip and Federated Learning* ${ }^{\star}$
}

\author{
István Hegedûs ${ }^{1[0000-0002-5356-2192]}$, Gábor Danner ${ }^{10000-0002-9983-1060]}$, and \\ Márk Jelasity ${ }^{1,2[0000-0001-9363-1482]}$ \\ 1 University of Szeged, Szeged, Hungary \\ 2 MTA SZTE Research Group on Artificial Intelligence, Szeged, Hungary
}

\begin{abstract}
Federated learning is a well-known machine learning approach over edge devices with relatively limited resources, such as mobile phones. A key feature of the approach is that no data is collected centrally; instead, data remains private and only models are communicated between a server and the devices. Gossip learning has a similar application domain; it also assumes that all the data remains private, but it requires no aggregation server or any central component. However - one would assume - gossip learning must pay a price for the extra robustness and lower maintenance cost it provides due to its fully decentralized design. Here, we examine this natural assumption empirically. The application we focus on is making recommendations based on private logs of user activity, such as viewing or browsing history. We apply low rank matrix decomposition to implement a common collaborative filtering method. First, we present similar algorithms for both frameworks to efficiently solve this problem without revealing any raw data or any user-specific parts of the model. We then examine the aggregated cost in both cases for several algorithm-variants in various simulation scenarios. These scenarios include a real churn trace collected over mobile phones. Perhaps surprisingly, gossip learning is comparable to federated learning in all the scenarios and, especially in large networks, it can even outperform federated learning when the same subsampling-based compression technique is applied in both frameworks.
\end{abstract}

\section{Introduction}

Mobile phones represent a key source of data and a very important platform not only for running pre-trained models but also for learning [17]. This is because collecting data centrally has become more and more problematic over the past

\footnotetext{
* In Springer CCIS 1167 (Proc. DMLE 2019). The final authenticated version is available online at https://doi.org/10.1007/978-3-030-43823-4_27. This study was supported by the Hungarian Government and the European Regional Development Fund under the grant number GINOP-2.3.2-15-2016-00037 ("Internet of Living Things") and by the Hungarian Ministry of Human Capacities (grant 203913/2018/FEKUSTRAT).
} 
few years due to novel data protection rules [7] as well as the increasing public awareness to privacy issues. For this reason, there is an increasing interest in methods that keep the raw data on the device and process it using distributed algorithms.

Google introduced federated learning to answer this challenge $[10,12]$. Not unlike the well-known parameter server architecture [6], a server maintains the current model and regularly distributes it to the workers who in turn calculate a gradient update and send it back to the server, where the updates are aggregated. In federated learning, this framework is optimized so as to minimize communication between the server and the workers. For this reason, the local update calculation is more thorough, and compression techniques can be applied when uploading the updates to the server. Gossip learning has also been proposed to address the same challenge $[9,14]$. This approach is fully decentralized, no parameter server is necessary. Nodes exchange and aggregate models directly. Since no infrastructure is required, and there is no single point of failure, gossip learning enjoys a significantly cheaper scalability and better robustness than centralized approaches.

However, it is not clear whether gossip learning is competitive in terms of convergence time and communication cost. To shed light on this question, we carry out an empirical comparison of the two approaches. To do this, we implement a recommender system in both paradigms, based on low-rank matrix decomposition. The gossip learning implementation is based on our previous work [9]. We propose a federated learning implementation as well, following the same design, but adapted to the centralized communication pattern. Also, inspired by [10], we apply subsampling to reduce communication in both approaches.

The result of our comparison is that gossip learning is in general comparable to the centrally coordinated federated learning approach, and in some scenarios it actually outperforms federated learning. One should obviously not jump to conclusions based on one empirical study, but our results suggest that fully decentralized algorithms perhaps deserve more attention in the future.

To sum up our key original contributions in the present study: (1) we propose an efficient collaborative filtering method for federated learning; (2) we improve several details of our previous solution [9] as well including the introduction of coordinate-based age parameters to manage aggregation and the application of an optimized version of subsampling to gossip learning; and (3) we compare the two methods empirically based on a realistic churn trace collected by the application Stunner [2].

We are aware of only two (at the time of writing, unpublished) studies that address the specific problem of recommender systems in federated learning. The first is based on the idea of meta-learning [4]. Here, it is assumed that the devices have enough data to learn a model based only on local data. Then, federated learning is used to find the optimal hyperparameters for the algorithm, using the devices to calculate gradients for the hyperparameters. We are interested in scenarios where there is not much local data, so meta-learning is not an option. The second study is closer to our approach [1] in spirit. However the authors as- 
sume a different setup with only implicit binary feedback as data (e.g., a movie was watched or not). Due to this, their input data is a dense matrix (there are no ratings labeled as "unknown") so compressed communication is more problematic. We focus on modeling only the known ratings (a small minority of all ratings) and make predictions based on these. Also, the optimization algorithm they approximate is alternating least squares with a federated gradient optimization step in the inner loop, while we use simple SGD which is more robust to failure and asynchrony.

We note that both approaches offer mechanisms for explicit privacy protection, apart from the basic feature of not collecting data. In federated learning, Bonawitz et al. [3] describe a secure aggregation protocol, whereas for gossip learning one can apply the methods described in [5]. Here, we are concerned only with the efficiency of the different communication patterns and do not compare security mechanisms.

The outline of the paper is as follows. In Section 2, we describe the low rank matrix decomposition problem, formulated as a machine learning problem. Here, we also present the key ideas to solve this problem in a decentralized setting. In Section 3, we describe the basics of solving the problem with federated learning while in Section 4 we present the gossip learning algorithm for the same problem. In Section 5 we present the key details of the learning algorithm that are common to both approaches. These include the details of the update rule, the subsampling technique, and the initialization. Finally, in Section 6, we present our empirical results.

\section{Rank- $k$ matrix approximation}

Here, we present the problem definition in the form of a model and a corresponding loss function. We also describe our approach and main assumptionscommon to both federated learning and gossip learning - regarding the optimization of the model.

\subsection{Problem definition}

The problem of rank- $k$ matrix approximation [11] is defined in the following way. Let $A \in \mathbb{R}^{m \times n}$ be a matrix that contains our data (for example, user ratings of items such as movies, songs, or locations). The goal is to find two matrices $X \in \mathbb{R}^{m \times k}$ and $Y \in \mathbb{R}^{n \times k}$ that minimize the error function

$$
J(X, Y)=\frac{1}{2}\left\|A-X Y^{T}\right\|_{F}^{2}=\frac{1}{2} \sum_{i=1}^{m} \sum_{j=1}^{n}\left(a_{i j}-\sum_{l=1}^{k} x_{i l} y_{j l}\right)^{2} .
$$

We consider the matrix $X Y^{T}$ an optimal rank- $k$ approximation of $A$. Note that the rank of $X$ and $Y^{T}$ (and therefore $X Y^{T}$ ) is at most $k$. Usually a $k$ much smaller than $m$ and $n$ is chosen to significantly compress the data. $X$ and $Y^{T}$ 
can be interpreted as high level features (e.g. genres of movies and tastes of users) that compactly represent the original data.

Often in practice we have only partial information regarding $A$; that is, some values in $A$ might be unknown. As an important generalization of the problem above, here we are looking for a rank- $k$ decomposition that approximates only the known values. A common approach is to minimize the error function

$$
J(X, Y)=\frac{1}{2} \sum_{(i, j) \in I}\left(a_{i j}-\sum_{l=1}^{k} x_{i l} y_{j l}\right)^{2}+\frac{\lambda}{2}\|X\|_{F}^{2}+\frac{\lambda}{2}\|Y\|_{F}^{2},
$$

where $I$ contains the indices of the known values of $A$. We can then use the decomposition $X Y^{T}$ to approximate the unknown values in $A$, since $X Y^{T}$ is fully defined. Note the here we also included additional regularization terms and the regularization parameter $\lambda$. This helps stabilize the optimization process in a machine learning context.

Another practical technique used is to add bias terms to the model. The bias terms $b \in \mathbb{R}^{m \times 1}$ and $c \in \mathbb{R}^{1 \times n}$ are incorporated into the model via the loss function

$$
J(X, Y, b, c)=\frac{1}{2} \sum_{(i, j) \in I}\left(a_{i j}-b_{i}-c_{j}-\sum_{l=1}^{k} x_{i l} y_{j l}\right)^{2}+\frac{\lambda}{2}\|X\|_{F}^{2}+\frac{\lambda}{2}\|Y\|_{F}^{2} .
$$

For example, in a recommender system, the bias can represent the fact that some users tend to give higher or lower scores than others, and some movies tend to get higher or lower scores. Intuitively, the bias represents average scores, and $X$ and $Y$ represent relative differences. This often enhances the prediction performance. With bias, the approximation of $A$ (both known and unknown values) is given by $X Y^{T}+b \mathbf{1}_{n}+c^{T} \mathbf{1}_{m}$ where $\mathbf{1}_{k}$ is a row vector of $k$ ones.

\subsection{Optimization approach}

Our targeted application environment consists of a potentially large set of personal devices holding private data. We follow the approach in our previous paper [9] and we will adapt the same approach to federated learning. We shall assume that each row in matrix $A$ is stored on exactly one device. We shall also assume that each device will host exactly one row. This setup covers applications where one row of the matrix belongs to one user and the devices belong to exactly one user, as in the case of mobile phones. One matrix row can naturally represent any kind of private user activity, such as watching movies. We should add though that if more than one row is stored on a device, the algorithms are still applicable.

The main idea is that matrix $X$ will also be stored in a similar manner; that is, every device will store the row of $X$ that belongs to the row of $A$ stored on the device. This way, the matrix $X$ that contains information about the users is completely private, every device knows only its own row. However, the entire 


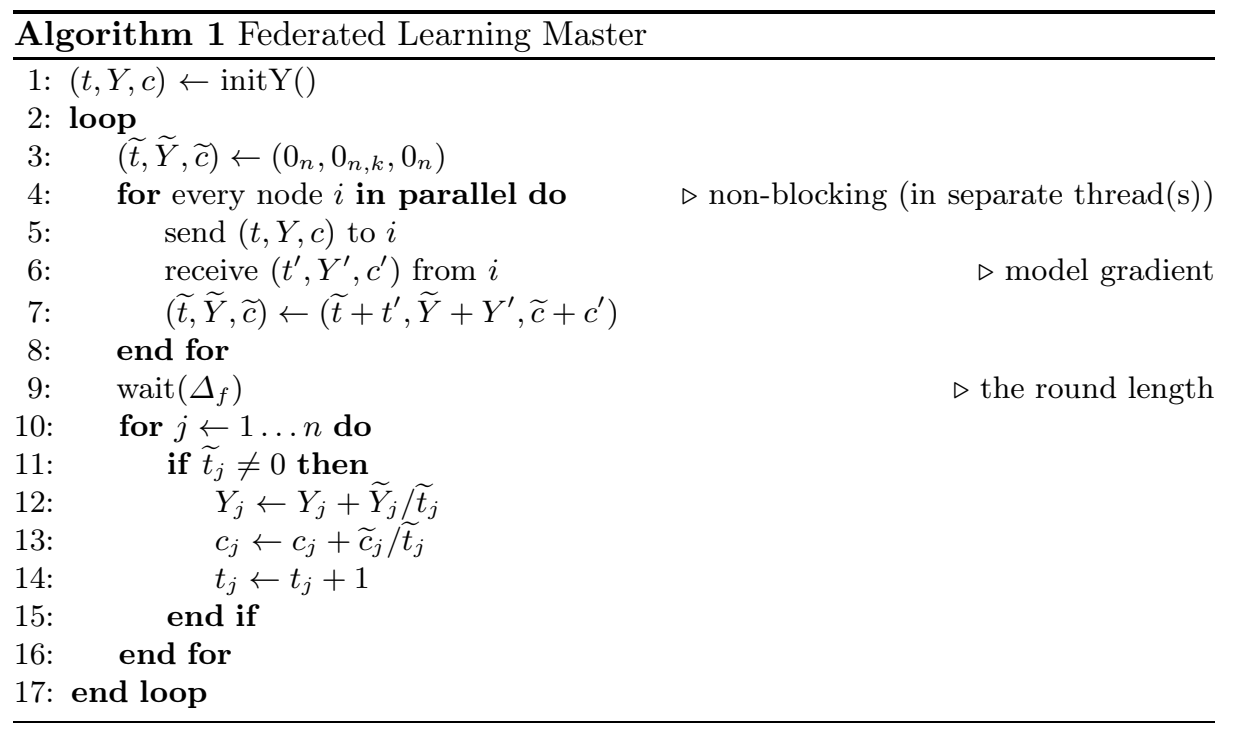

matrix $Y$ will be shared among all the devices. This is safe, because matrix $Y$ contains only user-independent information about all the items the users might consume.

The gradient of $Y$ computed by a single user may leak private data. In federated learning, Bonawitz et al. [3] describe a secure aggregation protocol with additional measures to prevent this kind of information leakage. In gossip learning, one can apply the secure distributed mini-batch methods described in [5]. We do not include such additional techniques in our present study.

Using the loss function defined in Equation (3), and assuming that every device has a copy of $Y$, the gradient of both its own row of $X$ and the global matrix $Y$ can be computed by each device locally, w.r.t. the local row of $A$. Devices can use these gradients to update their own row of $X$ locally. Therefore, all we need to take care of is to somehow aggregate the gradients of $Y$ over the devices and then redistribute new versions of $Y$. Federated learning and gossip learning offer two, rather different alternative solutions to this problem. (Note that the bias vectors $b$ and $c$ are handled similarly to $X$ and $Y$, respectively.)

\section{$3 \quad$ Federated Learning}

Here, we present the well-known federated learning algorithm [10,12], adapted to the problem of rank- $k$ matrix decomposition.

In this framework, there is a master node that runs Algorithm 1, and several worker nodes that execute Algorithm 2. The master first initializes the global model $(t, Y, c)$ that contains matrix $Y$, the bias vector $c$ and an age vector $t$. For each row $j, t_{j}$ counts how many times $Y_{j}$ and $c_{j}$ have been updated. Having a separate counter for each row is necessary because there can be a very different 


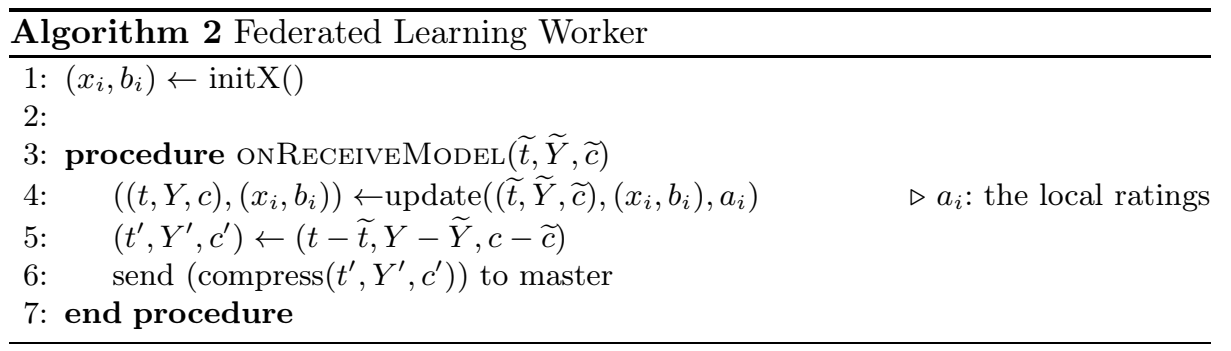

number of examples for each item, and thus there can be a different number of updates applied to each row (see below).

Similarly, each worker node initializes its private model $\left(x_{i}, b_{i}\right)$ that contains its own row of $X, x_{i}$, and the corresponding bias $b_{i}$. After initialization, in every round, the master sends the global model to all the workers. The workers then update the received global model and their own local user model using the local ratings, and they then send the (potentially compressed) model gradient to the master. In this message to the master, the vector $t^{\prime}$ can contain only ones and zeros, indicating which rows of $Y$ (and elements of $c$ ) were updated. At the end of each round, the server updates the global model with the average of the received gradients.

Each row $Y_{j}$ (and value $c_{j}$ ) will typically have different associated $\tilde{t}_{j}$ values depending on how many valid (non-missing) values are there in the matrix column $A_{j}$, and also on which clients manage to send a message to the master in the given round. We can think of $\widetilde{t}_{j}$ as the effective mini-batch size corresponding to updating $Y_{j}$ and $c_{j}$. Thus, by normalizing with $\widetilde{t}_{j}$, we effectively perform parallel mini-batch updates on $Y_{j}$ and $c_{j}$.

Note that in the federated learning framework it is typically assumed that the workers are synchronized; that is, the master has to wait until all (or most of) the nodes send a gradient in the given round and, most importantly, the workers have to wait as well for the next globally aggregated model from the master to process. Although asynchronous distributed learning is common, federated learning also seeks to handle the non-uniform sampling of training data, which is expected to make asynchronous implementations less stable.

The methods update, COMPRESS, InITX and INITY shall be explained in detail in Section 5. Note that the same methods are used in gossip learning as well.

\section{Gossip Learning}

In gossip learning, there is no master node. All the participants are equivalent, and form a P2P network [14]. All the nodes run Algorithm 3. The nodes first initialize their own copy of the global model $(t, Y, c)$ as well as the private model $\left(x_{i}, b_{i}\right)$. Then, in each cycle, they send their (potentially compressed) copy of the global model to a random online neighbor in the P2P network. Upon receiving a 

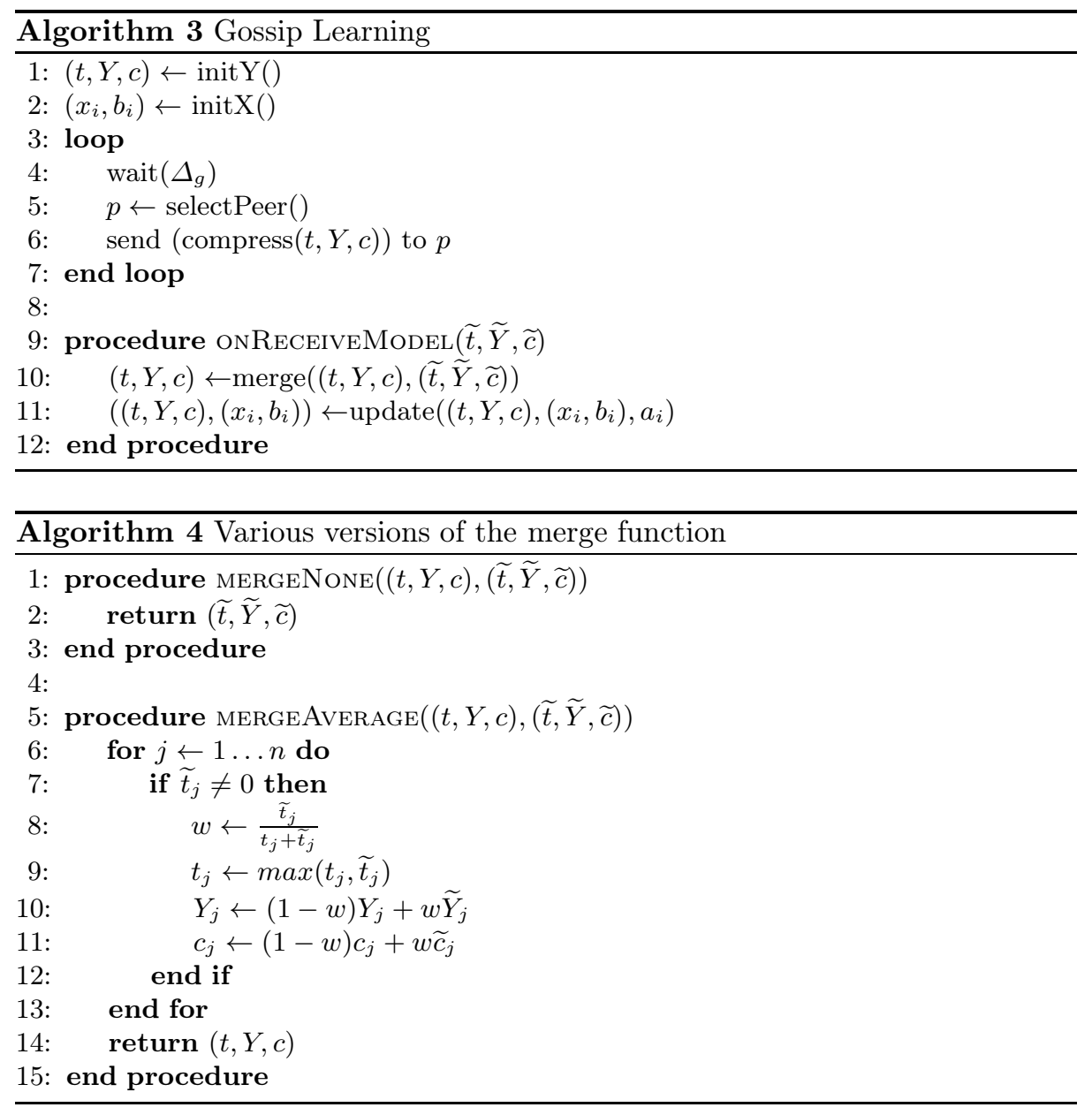

model, the node merges it into its own, then updates both the resulting merged new global model and the local model, using the local ratings.

As mentioned above, methods update, COMPREss, initX and InitY shall be explained in detail in Section 5. Note that the same methods are used by federated learning as well.

Method MERGE, however, is specific to gossip learning, and it is responsible for aggregating the updates computed at the devices. Possible implementations of this method are listed in Algorithm 4. The first option is not to perform any aggregation, in which case different versions of the global model perform random walks in the network independently. The other option is to take the average of the two models row by row, weighted by the corresponding elements of the age vectors so that the more converged copy has a larger effect.

An important effect of this weighted merging technique is that the freshly initialized rows of the model of any newly joined node are ignored. This is because 


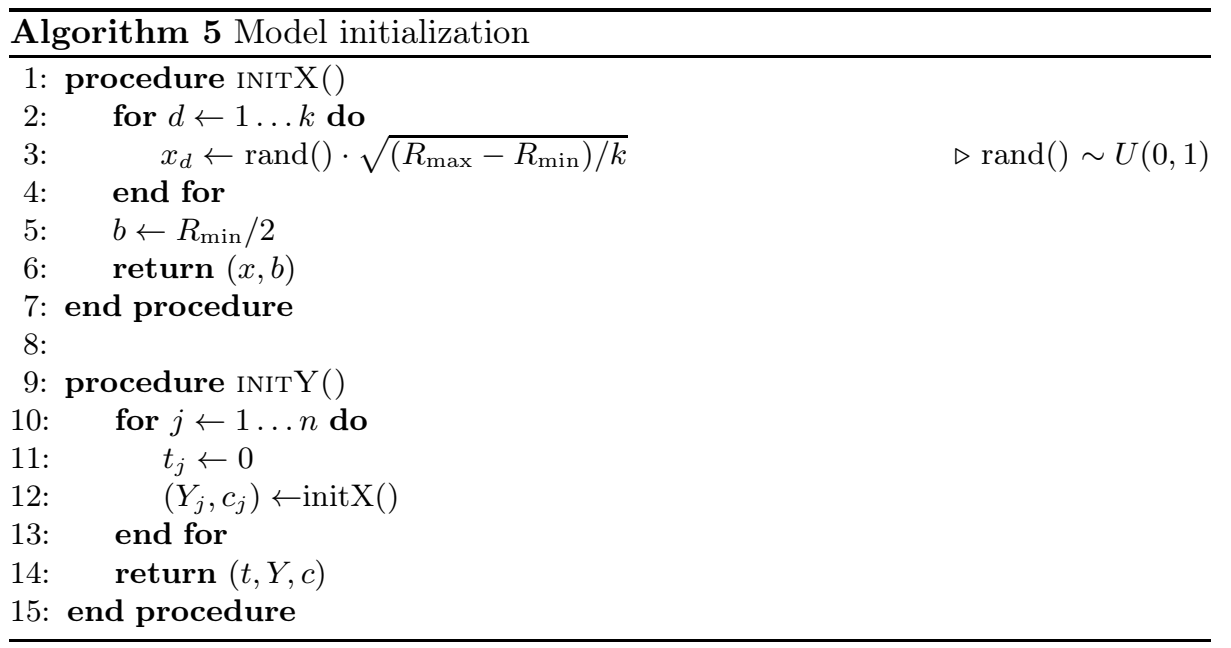

if a row has never been updated, then the age is zero for the given row. The new model will be assigned the maximum of the two merged ages. This is a conservative heuristic that performed better in our preliminary experiments than possible alternatives such as the sum of the two ages. Note that the age of the different rows can differ significantly because the number of known ratings for different items typically has a large variance.

Since gossip learning uses a P2P network, we have to make our assumptions about this network explicit. We assume that there is a membership service in our system. This service provides unique identities to the participants that might include public and private keys for public key cryptography that are tied to the network address of the node. The membership service also offers peer sampling, accessed through method SELECTPEer. That is, all the nodes are assumed to have access to addresses of live nodes from the network. In practice, peer sampling can have a decentralized implementation that can be dynamic [16] or it can be based on a static network with random neighbors [15] that is able to handle NAT devices as well. It can also be implemented as a centralized service. Ideally, the neighbors returned by the peer sampling service should be uniform random samples of the live nodes, but in practice it suffices if the network has good mixing when, for example, the neighbors are sampled from a fixed overlay network graph.

\section{Shared Methods}

Here, we present those methods that are used by both federated learning and gossip learning. Let us begin with the initialization methods in Algorithm 5. Both $X$ and $Y$ are initialized with uniform random numbers from the range $\left[0, \sqrt{\left(R_{\max }-R_{\min }\right) / k}\right]$, and the initial bias is set to $R_{\min } / 2$, where $R_{\max }$ and $R_{\text {min }}$ are the largest and the smallest possible ratings, respectively. This ensures 


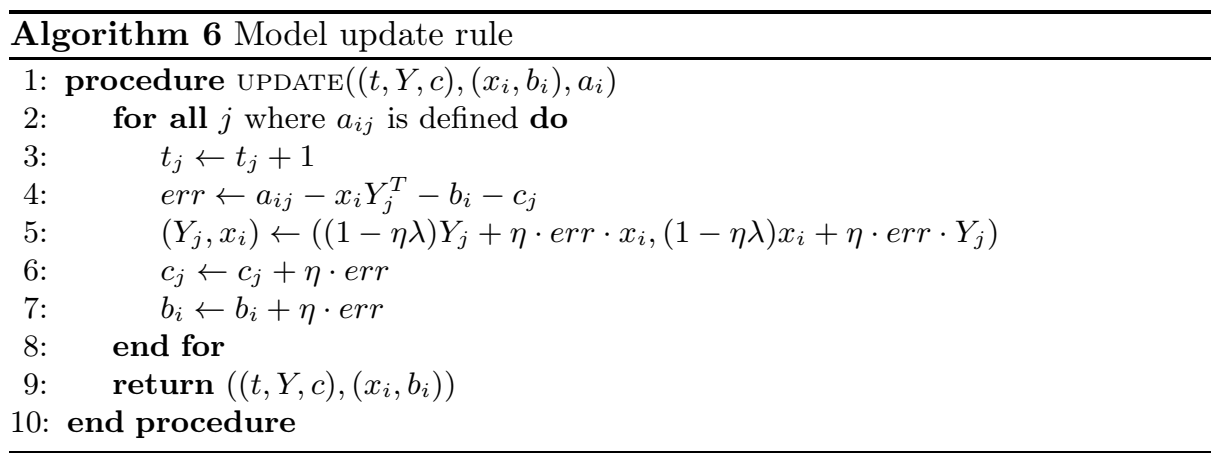

that when a prediction $x_{i} Y_{j}^{T}+b_{i}+c_{j}$ is made using initial values, the result falls in the range $\left[R_{\min }, R_{\max }\right]$.

As for learning, both models use a stochastic gradient descent (SGD) update rule with the fixed learning rate $\eta$ (see Algorithm 6). The age vector $t$ is incremented in positions corresponding to updated rows of $Y$ (that is, for those items that the user rated). The update rule simply follows from the partial derivatives of (3). Note that this version of the update rule uses a constant learning rate, but other implementations might also use the age vector passed to the update method.

Let us now turn to the compression methods. In this study, we focus on subsampling as a simple compression technique. That is, only $s$ rows of $Y$ are sent along with the corresponding elements of $t$ and $c$, where $s$ is the compression parameter (see Algorithm 7). Subsampling is performed randomly without replacement from the updated rows (that is, those rows where the corresponding rating is known) and, if there is still room left, from the remaining, non-updated rows. Note that sending non-updated rows in fact makes sense because in such cases the given node might act as a forwarding agent. In other words, such rows might be useful for the recipient nodes.

\section{Experiments}

Here, we present our simulation experiments with gossip learning and federated learning over the MovieLens database in several scenarios.

\subsection{Datasets}

The MovieLens data sets [8] were collected by the GroupLens Research Project at the University of Minnesota. The data was collected through the MovieLens website (movielens.org) over various periods of time, depending on the size of the set. The main properties of the MovieLens data sets are shown in Table 1 and Figure 1. Each data set is split into a training matrix and a test matrix in such a way that for each user, there are either 0 or 10 defined values in the 


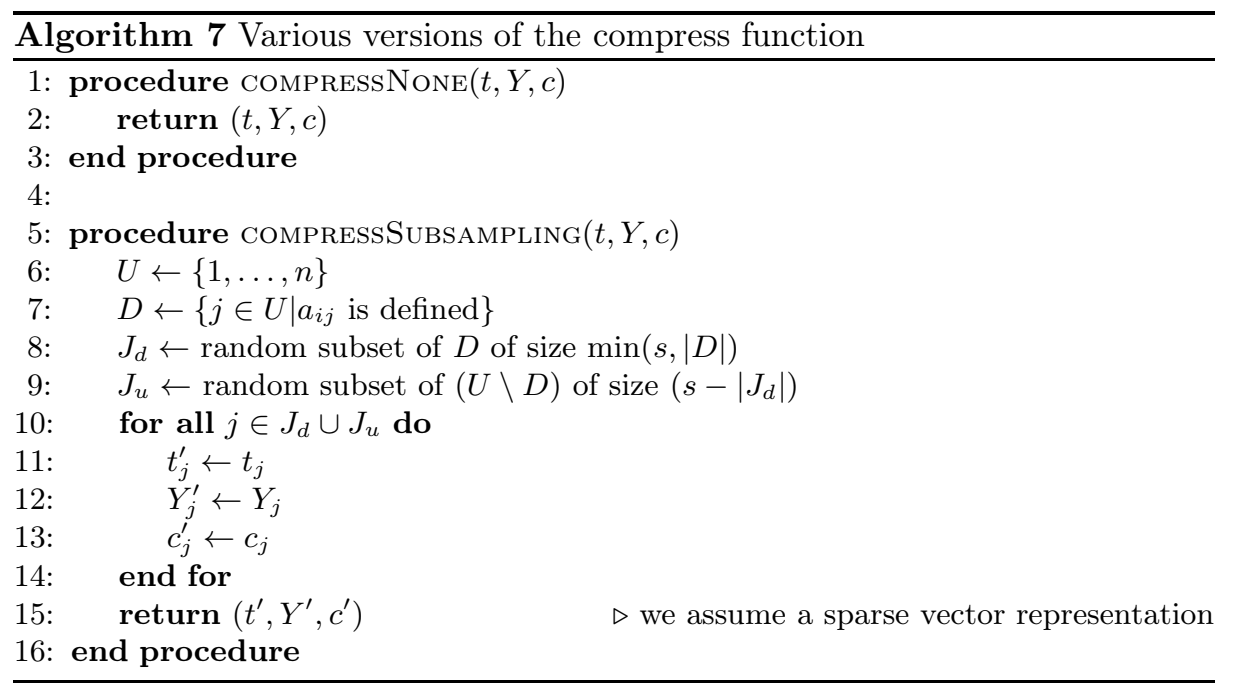

Table 1. The main properties of the MovieLens data sets and algorithm parameters

\begin{tabular}{|l||c|c|c|}
\hline & $100 \mathrm{~K}$ & $1 \mathrm{M}$ & $10 \mathrm{M}$ \\
\hline \hline$\#$ users $(m)$ & 943 & 6,040 & 69,878 \\
\hline$\#$ movies $(n)$ & 1,682 & 3,952 & 10,677 \\
\hline$\#$ ratings & 100,000 & $1,000,209$ & $10,000,054$ \\
\hline Density & $6.3 \%$ & $4.2 \%$ & $1.3 \%$ \\
\hline Training / Test & $90.57 \% / 9.43 \%$ & $93.96 \% / 6.04 \%$ & $93.01 \% / 6.99 \%$ \\
\hline Time period & $20.09 .97-22.04 .98$ & $25.04 .00-28.02 .03$ & $09.01 .95-05.01 .09$ \\
\hline \hline$\eta / \lambda / k$ & $10^{-2} / 10^{-1} / 5$ & $10^{-2} / 10^{-1} / 5$ & $10^{-2} / 10^{-1} / 5$ \\
\hline message size & $0.6 \mathrm{Mbit}$ & $1.5 \mathrm{Mbit}$ & $4.1 \mathrm{Mbit}$ \\
\hline
\end{tabular}

test matrix. Each row of the training matrix (representing the ratings of a given user) was assigned to a unique node in the simulation experiments.

\subsection{System Model}

In our simulations, fixed random 20-out graphs were used as the overlay network. The number of nodes was equal to the number of users in the given data set. In the churn-free scenario, every node stayed online for the whole experiment. A real availability trace, gathered from smartphones, was used in the churn scenario. A message was considered successfully delivered if and only if both the sender and the receiver remained online during the transfer. Peer selection (method SELECTPEeR) returned online nodes only.

The nodes had the same upload and download bandwidths. The motivation for this was that it is likely that in a real application there will be a low, uniform, 


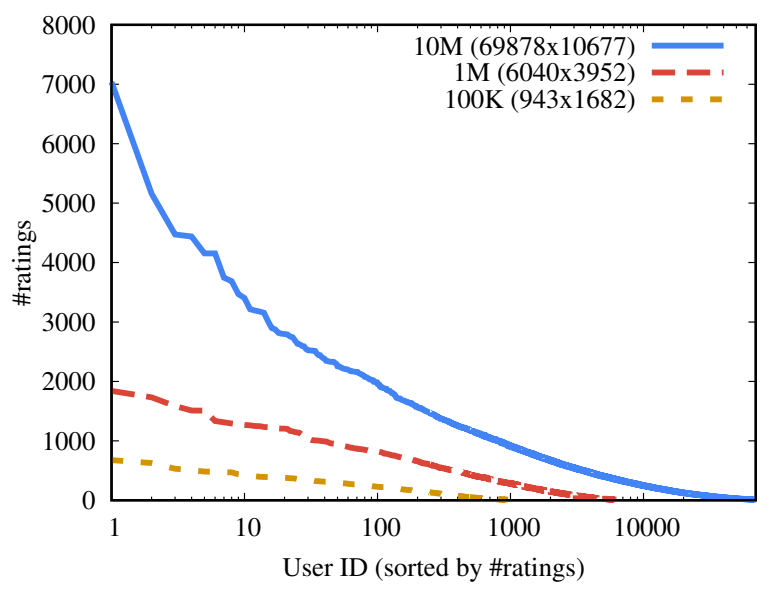

Fig. 1. Visualization of the distribution of the number of rated items per user. Users are sorted according to the number of their rated items for all three databases.

configured bandwidth cap. The server had infinite bandwidth (which favors federated learning, as gossip learning does not use a server). The transfer time of a full model was assumed to be $1728 \mathrm{~s}$ (irrespective of the data set used) in the low bandwidth scenario, and $172.8 \mathrm{~s}$ in the high bandwidth scenario. This allowed for around 100 and 1000 iterations over the course of 48 hours, respectively.

The cycle length parameters $\Delta_{g}$ and $\Delta_{f}$ were set so that the two approaches fully utilized the available bandwidth. In our case this also means that the two algorithms transfer the same amount of data overall in the network in the same amount of time, making comparisons of convergence dynamics fair. The gossip cycle length $\Delta_{g}$ is exactly the transfer time of a model, which is proportionally smaller when compression is used. The cycle length $\Delta_{f}$ of federated learning is the round-trip time, that is, the sum of the upload and download times. In this case, only the upstream transfer is compressed.

Note that we use rather low bandwidth settings because in the churn scenario if the transfer is very fast, the network hardly changes during the learning process, the models are learned over an effectively static subset of the nodes. Slower transfer is more challenging, because more transfers fail, just like in the case of very large machine learning models such as deep neural networks. (This issue is completely irrelevant in the churn-free scenario, since the dynamics are identical apart from the scale of time.)

\subsection{Smartphone Traces}

We used a trace collected by STUNner, a locally developed, openly available smartphone application [2]. In short, the app monitors and collects information about the battery level, charging status, bandwidth, and NAT type. 

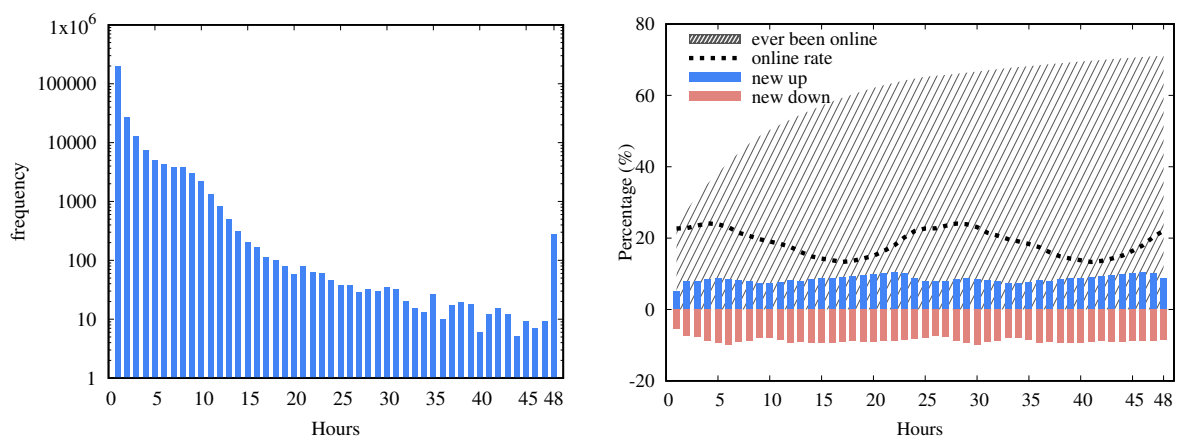

Fig. 2. Online session length histogram (left) and device churn (right).

The trace contains time series spanning varying lengths of time, originating from 1191 different users. Based on the UTC hour of day, we split the data into 2-day segments (with a one-day overlap), resulting in 40,658 segments altogether. Using this, we can simulate a virtual 48-hour period by assigning a segment to each simulated node.

To make our algorithm phone and user friendly, we consider a device to be online (available) when it has been on a charger and connected to the Internet (with a bandwidth of at least $1 \mathrm{Mbit} / \mathrm{s}$ ) for at least a minute, therefore we do not use battery power at all.

The main properties of the trace are shown in Figure 2. The plot on the right illustrates churn by showing what percentage of the nodes left, or joined the network (at least once) in any given hour. Notice that at any given moment about $20 \%$ of the nodes are online. The mean online session length is $81.368 \mathrm{~min}$.

\subsection{Hyperparameters}

The learning rate $\eta$ and regularization parameter $\lambda$ were optimized in the churnfree, low-bandwidth, uncompressed scenario. The resulting values are $\eta=10^{-2}$ and $\lambda=10^{-1}$, as shown in Table 1 . We used rank-5 factorization.

\subsection{Results}

We used PeerSim [13] for the simulations. We measured performance with the help of the root-mean-square deviation

$$
\mathrm{RMSE}=\sqrt{\frac{1}{|T|} \sum_{i, j \in T}\left(r_{i, j}-x_{i} y_{j}^{T}\right)^{2}},
$$

where $R \in \mathbb{R}^{m \times n}$ is the test matrix, and $T$ is the set of indices defined in $R$. In the case of gossip learning, the error is calculated using the models stored in the currently online nodes and the corresponding rows of $R$. In the case of federated 

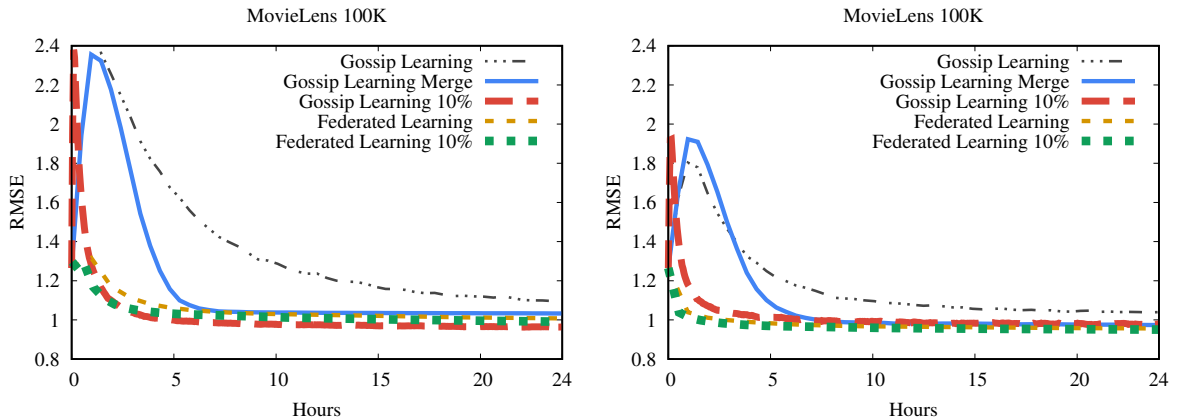

MovieLens 1M

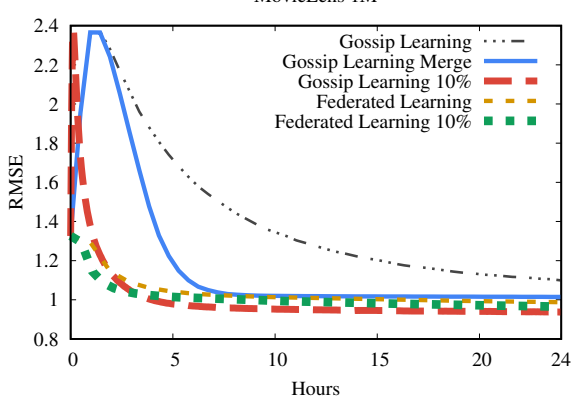

MovieLens 1M

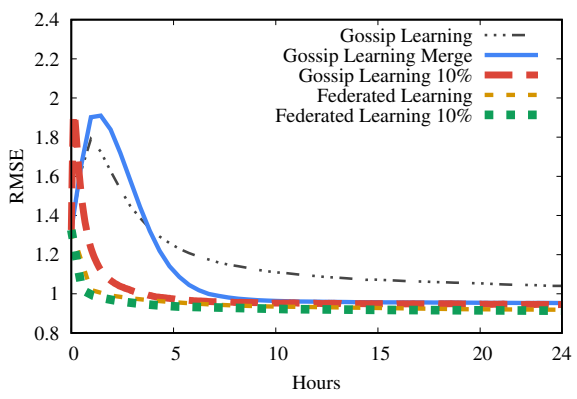

MovieLens 10M
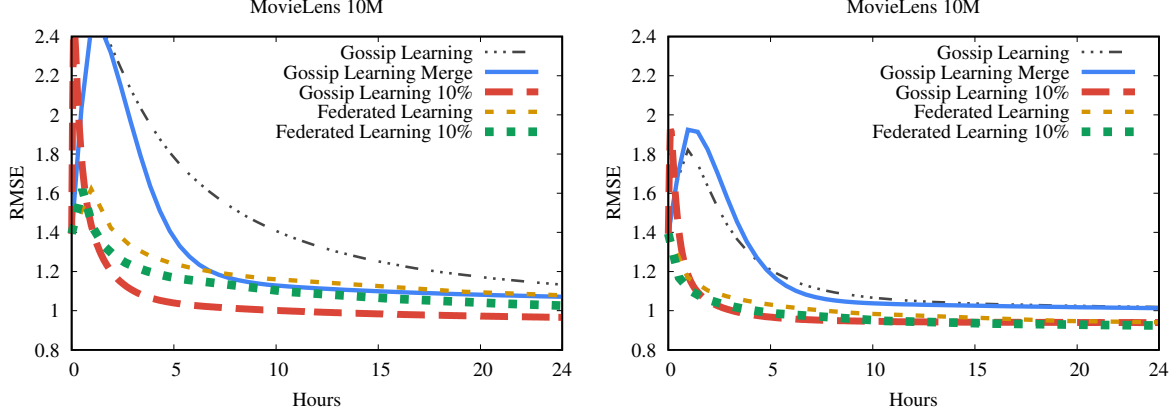

Fig. 3. Churn-free scenario with 1 epoch (left) and 10 epochs (right).

learning, the aggregated global model is used instead of the local ones. Figure 3 contains our results without churn, and Figure 4 shows the same experiments over the smartphone trace. The evaluated algorithms are

Gossip Learning: no merging and no subsampling. Here, the cycle length equals the time needed for one full model transmission.

Gossip Learning Merge: with merging but no subsampling, so the cycle length is still one full transmission.

Gossip Learning 10\%: with merging and subsampling with $s=n / 10$. Here, the cycle length corresponds to 0.1 full transmissions.

Federated Learning: no subsampling, so the cycle length corresponds to two full transmissions: upload and download. 
Federated Learning 10\%: the uploaded model is subsampled with $s=n / 10$, so the cycle length corresponds to 1.1 full transmissions.

In Figure 3 we include results with 1 and 10 epochs of local learning in the left and right columns, respectively. In the case of 10 epochs, the local gradient update step is iterated 10 times. Clearly, for both methods, increasing the number of epochs improves convergence speed without any extra communication. The compressed variants consistently perform better. Federated learning has an initial advantage, which disappears after a few hours. In fact, for the largest problem, gossip learning is almost identical to federated learning, and the difference between the two methods seems to decrease with increasing network size. Interestingly, when only 1 epoch is performed, gossip learning actually outperforms federated learning by a significant margin especially on the largest network.

In Figure 4 we ran one local epoch in each experiment, but the plots on the right show the effect of speeding up communication. Faster communication results in a dramatically better performance, simply because the convergence speed is able to "beat" the speed of churn. Apart from this observation, the other conclusions are similar, namely compression helps both methods and gossip learning performs relatively better in larger networks. Overall, federated learning and gossip learning have a very similar performance, despite the disadvantage of gossip learning of not relying on a central server for aggregation and broadcast.

\section{Conclusions}

In this study, our main goal was to explore the differences between federated learning and gossip learning over a collaborative filtering task. Since gossip learning does not rely on central servers, one might expect it to pay a performance penalty in terms of convergence speed, when given the same communication budget.

Our main conclusion based on our empirical study is that federated learning does not seem to have a clear performance advantage. In fact, in certain scenarios gossip learning proved to be preferable. Obviously, the design space for both protocols is very large, and there are many possibilities for improving the communication efficiency in both paradigms. It is also a non-trivial question of how one should model communication constraints and costs, since this depends on many factors. However, it is interesting, and perhaps non-trivial, that gossip learning is clearly comparable in performance. This might motivate further research into fully decentralized methods that otherwise have clear benefits such as a very low cost of entry that is not dependent of the network size, or the robustness due to the lack of any critical components.

\section{References}

1. Ammad-ud-din, M., Ivannikova, E., Khan, S.A., Oyomno, W., Fu, Q., Tan, K.E., Flanagan, A.: Federated collaborative filtering for privacy- 

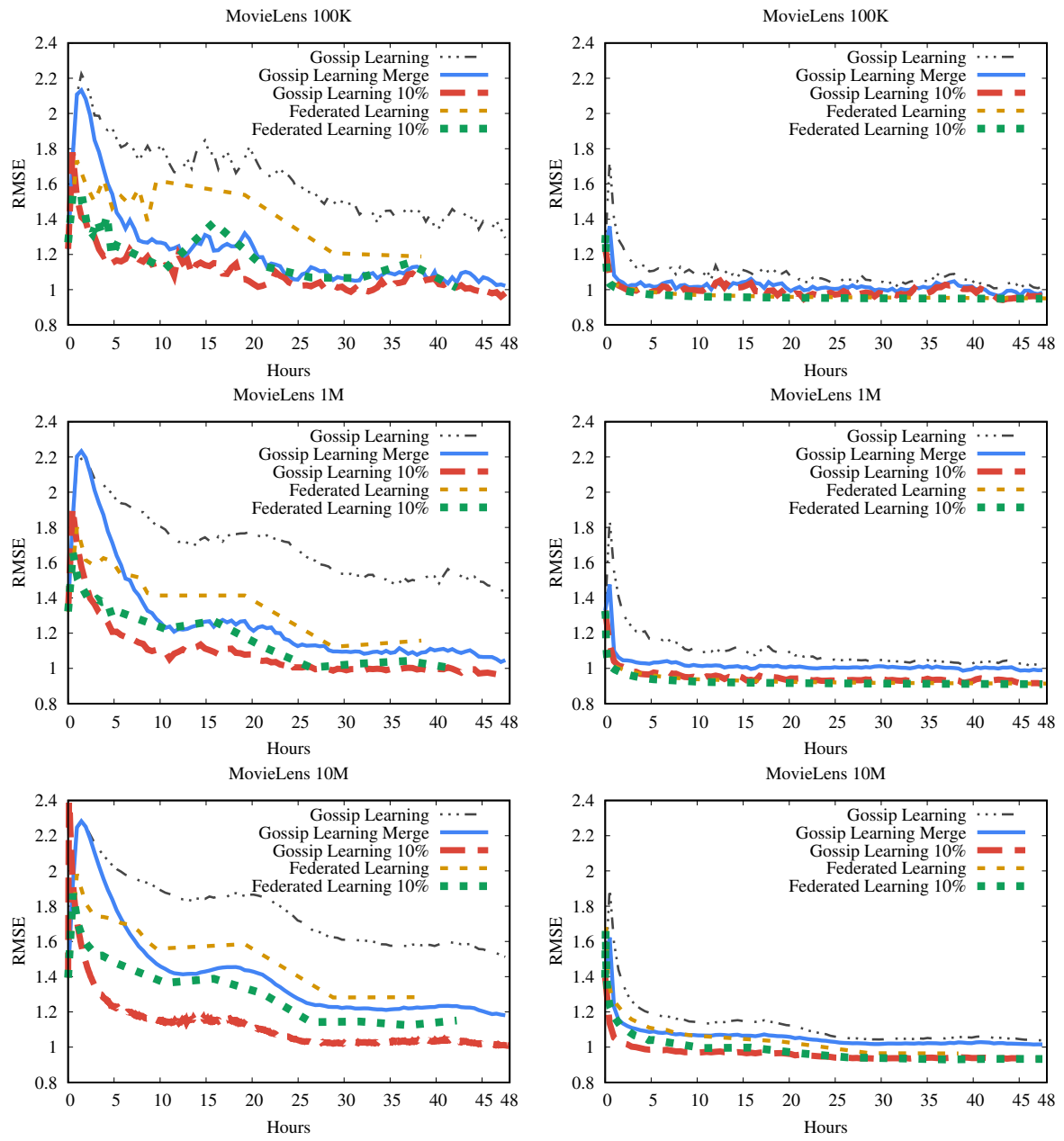

Fig. 4. Churn trace scenario with low bandwidth (left) and $10 \times$ higher bandwidth (right).

preserving personalized recommendation system. CoRR abs/1901.09888 (2019), http://arxiv.org/abs/1901.09888

2. Berta, Á., Bilicki, V., Jelasity, M.: Defining and understanding smartphone churn over the internet: a measurement study. In: Proceedings of the 14th IEEE International Conference on Peer-to-Peer Computing (P2P 2014). IEEE (2014). https://doi.org/10.1109/P2P.2014.6934317

3. Bonawitz, K., Ivanov, V., Kreuter, B., Marcedone, A., McMahan, H.B., Patel, S., Ramage, D., Segal, A., Seth, K.: Practical secure aggregation for federated learning on user-held data. In: NIPS Workshop on Private Multi-Party Machine Learning (2016)

4. Chen, F., Dong, Z., Li, Z., He, X.: Federated meta-learning for recommendation. CoRR abs/1802.07876 (2018), http://arxiv.org/abs/1802.07876 
5. Danner, G., Berta, Á., Hegedús, I., Jelasity, M.: Robust fully distributed minibatch gradient descent with privacy preservation. Security and Communication Networks 2018, 6728020 (2018). https://doi.org/10.1155/2018/6728020

6. Dean, J., Corrado, G.S., Monga, R., Chen, K., Devin, M., Le, Q.V., Mao, M.Z., Ranzato, M., Senior, A., Tucker, P., Yang, K., Ng, A.Y.: Large scale distributed deep networks. In: Proceedings of the 25th International Conference on Neural Information Processing Systems - Volume 1. pp. 1223-1231. NIPS'12, Curran Associates Inc., USA (2012), http://dl.acm.org/citation.cfm?id=2999134.2999271

7. European Commission: General data protection regulation (GDPR) (2018), https://ec.europa.eu/commission/priorities/justice-and-fundamental-rights/dataprotection/2018-reform-eu-data-protection-rules

8. Harper, F.M., Konstan, J.A.: The movielens datasets: History and context. ACM Trans. Interact. Intell. Syst. 5(4), 19:1-19:19 (Dec 2015). https://doi.org/10.1145/2827872, http://doi.acm.org/10.1145/2827872

9. Hegedús, I., Berta, Á., Kocsis, L., Benczúr, A.A., Jelasity, M.: Robust decentralized low-rank matrix decomposition. ACM Transactions on Intelligent Systems and Technology 7(4), 62:1-62:24 (May 2016). https://doi.org/10.1145/2854157

10. Konecný, J., McMahan, H.B., Yu, F.X., Richtárik, P., Suresh, A.T., Bacon, D.: Federated learning: Strategies for improving communication efficiency. In: Private Multi-Party Machine Learning (NIPS 2016 Workshop) (2016)

11. Koren, Y., Bell, R., Volinsky, C.: Matrix factorization techniques for recommender systems. Computer 42(8), 30-37 (2009). https://doi.org/10.1109/MC.2009.263

12. McMahan, B., Moore, E., Ramage, D., Hampson, S., y Arcas, B.A.: Communication-efficient learning of deep networks from decentralized data. In: Singh, A., Zhu, J. (eds.) Proceedings of the 20th International Conference on Artificial Intelligence and Statistics. Proceedings of Machine Learning Research, vol. 54, pp. 1273-1282. PMLR, Fort Lauderdale, FL, USA (20-22 Apr 2017)

13. Montresor, A., Jelasity, M.: Peersim: A scalable P2P simulator. In: Proceedings of the 9th IEEE International Conference on Peer-to-Peer Computing (P2P 2009). pp. 99-100. IEEE, Seattle, Washington, USA (Sep 2009). https://doi.org/10.1109/P2P.2009.5284506, cikkek/p2p09.pdf, extended abstract

14. Ormándi, R., Hegedûs, I., Jelasity, M.: Gossip learning with linear models on fully distributed data. Concurrency and Computation: Practice and Experience 25(4), 556-571 (2013). https://doi.org/10.1002/cpe.2858

15. Roverso, R., Dowling, J., Jelasity, M.: Through the wormhole: Low cost, fresh peer sampling for the internet. In: Proceedings of the 13th IEEE International Conference on Peer-to-Peer Computing (P2P 2013). IEEE (2013). https://doi.org/10.1109/P2P.2013.6688707

16. Tölgyesi, N., Jelasity, M.: Adaptive peer sampling with newscast. In: Sips, H., Epema, D., Lin, H.X. (eds.) Euro-Par 2009. Lecture Notes in Computer Science, vol. 5704, pp. 523-534. Springer-Verlag (2009). https://doi.org/10.1007/978-3-64203869-3_50

17. Wang, J., Cao, B., Yu, P.S., Sun, L., Bao, W., Zhu, X.: Deep learning towards mobile applications. In: IEEE 38th International Conference on Distributed Computing Systems (ICDCS). pp. 1385-1393 (Jul 2018). https://doi.org/10.1109/ICDCS.2018.00139, https://arxiv.org/pdf/1809.03559.pdf 